\title{
X-ray Absorption Near Edge Structure Studies of Transition Metal Complexes of O-Phenylenediamine and Substituted Aniline Ligand
}

\author{
Kamaljeet S. Sura ${ }^{*}$, A. Mishra ${ }^{2}$, S. Patidar ${ }^{3}$, S. Mohammad ${ }^{4}$ \\ ${ }^{1}$ School of Physics, DAVV, Indore (M.P), India \\ ${ }^{2}$ School of Physics, DAVV, Indore (M.P), India \\ ${ }^{3}$ School of Physics, DAVV, Indore (M.P), India \\ ${ }^{4}$ School of Physics, DAVV, Indore (M.P), India \\ *Corresponding Author: kamaljeetsura@gmaill.com, Tel.: +91-99070-53818
}

Available online at: www.isroset.org

Received: 13/May/2018, Revised: 21/May/2018, Accepted: 07/Jun/2018, Online: 30/Jun/2018

\begin{abstract}
The present paper deals with the synthesis of transition metal complexes of Copper by chemical rout method. The complexes have been synthesized by condensation of o-phenylenediamine, diethyl malonate and diazonium ion in the ethanolic medium, through refluxing with $\mathrm{Cu}$ (II) metal salt. Synthesized metal complexes were characterized by X-ray diffraction (XRD) and studied by X-ray absorption structure (XAS) spectroscopy. The XAS technique includes XANES (X-ray Absorption near Edge structure) and EXAFS (Extended X-ray Absorption Fine Structure) spectroscopy. XANES spectra have been recorded at the $\mathrm{K}$-edge of $\mathrm{Cu}$ using the dispersive EXAFS (DEXAFS) beam line at $2.5 \mathrm{GeV}$ Indus-2 synchrotron radiation source RRCAT, Indore, India. The XANES data have been analysed using the computer software Athena. These have been used to determine the chemical shift, edge width, and nature of complexes. XRD analysis shows that sample is crystalline in nature. The XRD pattern is made by using Bruker D-8 advance instrument.
\end{abstract}

Keywords-XRD, XANES, chemical shift,edge width

\section{INTRODUCTION}

The transition metal (Copper) complexes has been extensively studied [1] and some of these complexes already have some interesting applications, e.g. in catalytic oxidation reactions and electrochemical reduction processes [2], in metalloenzyme-mediated catalysis $[3,4]$ and asymmetric catalysis, metal-containing liquid-crystalline polymers [5], as catalytically active materials to develop surface-modified electrodes [6,7]. The Msalen type chelates have attracted considerable interest due to their ability to bind reversibly $\mathrm{O}_{2}$ and $\mathrm{CO}_{2}[1,3,8]$, and because of that they can be used as "metalloligands" for design of a various homo- or heterodinuclear complexes [9]. It has been reported that metal complexation alters the various physiological properties especially the cytotoxic and antitumor activities of many naturally occurring compounds [10] it also shows antifungal potential against Alternaria alternate, Fusarium and Myrothecium roridum by inhibiting spore germination [11]. It is also demonstrated that the coordination of metal ions with bioactive ligands can actually improve the pharmaceutical activity of drugs $[12,13]$.

X-ray absorption spectroscopy (XAS) has been revealed to be a best tool for determining the electronic and geometrical structure of complex compounds. XANES reveal to identify the allowed transitions and also the mixing or splitting of the final state orbital. Using known structural data, informative deductions on structure-bonding relations have been made. Since the spectra can be influenced by the state of the $d$ orbital, because it is primarily responsible for the bonding with ligands. For $\mathrm{Cu}$ containing complexes, $\mathrm{Cu}$ K-edge XANES is use to derive information on the electronic and geometrical structure [14].

\section{METHODOLOGY}

1. Chemicals and solvent: All chemicals (sodium nitrite, sodium acetate, O-phenylenediamine, diethyl malonate, ferric chloride and anilines) are of Loba Chemie and solvents (absolute ethyl alcohol) are of Loba Chemie all are laboratory reagent grades and were used without further purifications.

2. Synthesis of Ligand In a $100 \mathrm{cc}$ beaker pertinent aniline derivative $(0.01 \mathrm{M})$ were dissolved in a mixture of $5.0 \mathrm{ml}$ of concentrated hydrochloric acid and $5.0 \mathrm{ml}$ of distilled water. Now the contents kept at freezing temperature in a deep freezer. To this, an aqueous solution of sodium nitrite $(0.01$, $1.38 \mathrm{~g}$ ) in $5.0 \mathrm{ml}$ of distill water was added drop wise with continuous stirring keeping the temperature of the reaction in the vicinity of $0-5^{\circ} \mathrm{C}$. Meanwhile, in another beaker, the 
particular diethylmalonate $(0.01 \mathrm{M})$, sodium acetate $(7.0 \mathrm{~g})$ and ethyl alcohol $(25 \mathrm{ml})$ were taken, mixed and cooled in an ice bath. Now the diazotized solution was added to this solution in a drop wise manner thorough stirring and maintaining the temperature of the contents below $5^{\circ} \mathrm{C}$. The reaction mixture was kept for overnight period, filtered through suction, washed with plenty of water and dried in vacuum. Fine crystals of coupled products were obtained in good yield. Structures of ligands are shown in following figure.

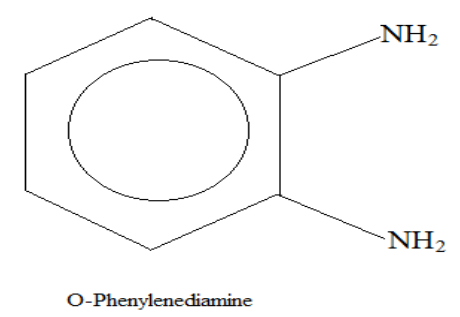

Ligand 1<smiles>CCOC(=O)C(=NNc1ccccc1C)C(=O)OCC</smiles>

diethyl 2-(2-o-tolylhydrazono)malonate Ligand 2(a)<smiles>CCOC(=O)C(=NNc1ccc(Cl)cc1)C(=O)OCC</smiles>

diethyl 2-(2-(4-chlorophenyl)hydrazono)malonate Ligand 2 (b)<smiles>CCOC(=O)C(=NNc1ccccc1[N+](=O)[O-])C(=O)OCC</smiles>

diethyl 2-(2-(2-nitrophenyl)hydrazono)malonate Ligand 2 (c)

Figure1. Structure of ligand and name.

3. Synthesis of complexes: In a $250 \mathrm{ml}$ round bottom flask equimolar ethanol solution of ligands and metal salt were taken and this mixture was refluxed for 4 hours. After reflux Solvent was evaporated to afford fine crystal of metal complexes. This was recrystallized from ethanol.

Formation of the metal complexes can be represented by the following general equations $\mathrm{MX}_{2}+2 \mathrm{~L} \rightarrow \mathrm{M}(\mathrm{L})_{2}+2 \mathrm{X}$. In this equation, $\mathrm{M}$ is $\mathrm{Cu}$ (II) metal, 2L is (Ligand 1 and Ligand 2).

\section{Structure of complex}

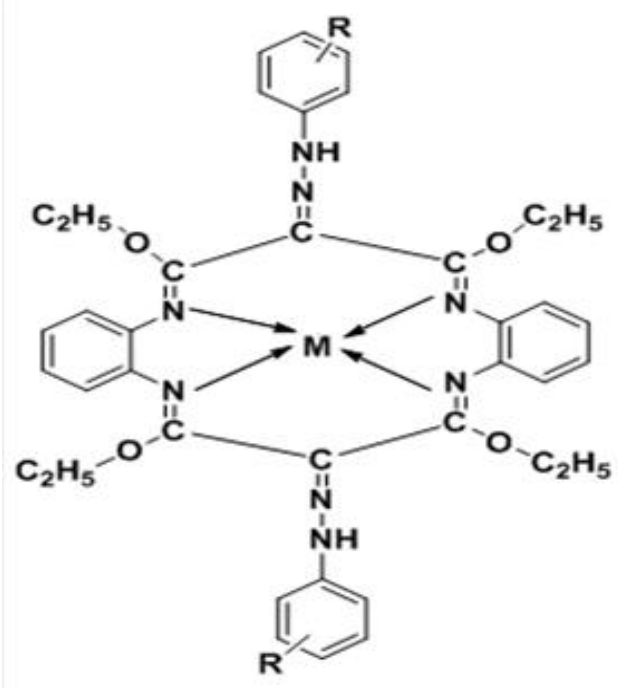

Figure2. Structure of transition metal complex, $\mathrm{M}$ is Copper (II) and $\mathrm{R}$ is substituted anilines.

4. X-ray Diffraction: The X-ray powder diffraction patterns of the complexes are indicative of their crystalline nature. The diffraction patterns have been successfully indexed. The X-ray analysis reveals that all the samples are in single phase. The XRD peaks of the single phase of both samples were indexed 
and particle size were calculated and presented in table 1 . The $2 \theta$ values obtained from the positions of Bragg peaks have been used to obtain lattice parameter. The XRD pattern is made by using Bruker D-8 advance instrument, at IUC Indore. 5. X-ray Absorption near Edge structure (XANES): X-ray absorption fine structure studies were carried out on beamline BL-8 dispersive EXAFS beamline at the 2.5GeV INDUS-2 Synchrotron Source, Raja Ramanna Centre for Advanced Technology (RRCAT), Indore, India. The intensity of transmitted radiation is recorded by position sensitive detector. The recorded data have been analysed using software Origin 8 and Athena 0.80 .056

\section{RESULTS AND DISCUSSION}

The diffraction pattern of complexes recorded between $2 \theta$ ranging from $10^{\circ}$ to $80^{\circ}$. The crystalline size of the samples is estimated using the Scherrer's formula. According to Scherrer's equation, the grain size is given by $\mathrm{t}=0.9 \lambda / \mathrm{B} \operatorname{Cos} \theta$, where $t$ is the crystal thickness, $B$ is half width (in radians) of the diffraction the line, $\theta$ is the Bragg angle and $\lambda$ is the wavelength. The grain size corresponding to each diffraction maxima can be determined from the measurement of the half width of the diffraction peak. The diffraction patterns have been successfully indexed. The value of the crystalline size is shown in table 1 . The crystalline size was found to be within in the range $32.61-52.87 \mathrm{~nm}$. It has been observed that crystalline size is different for every sample due to change in the $\mathrm{R}$ (substituted aniline) position.

The profiles of the K-absorption discontinuity of three copper complexes are shown in figure 4 . In present observation, The K-absorption edge of copper has been found to be shifted towards the high energy side in all the complexes studied as compared to the K-absorption edge in the metal. The shifts of the K-absorption edge of copper in the complexes with respect to that of copper metal have been determined.

Figures and Tables

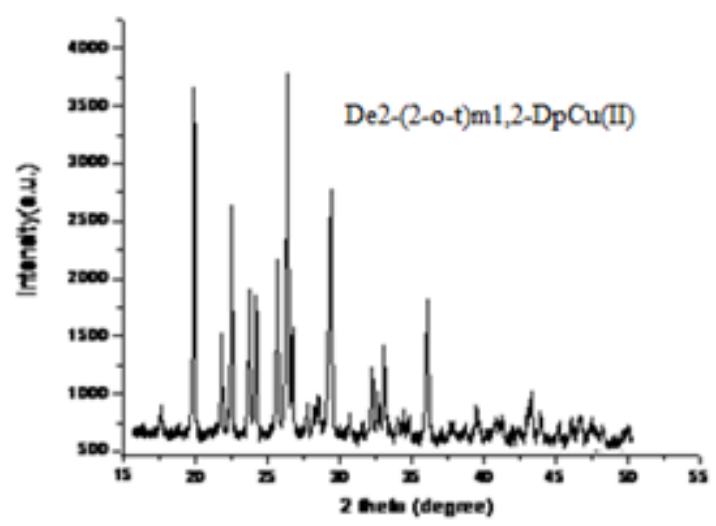

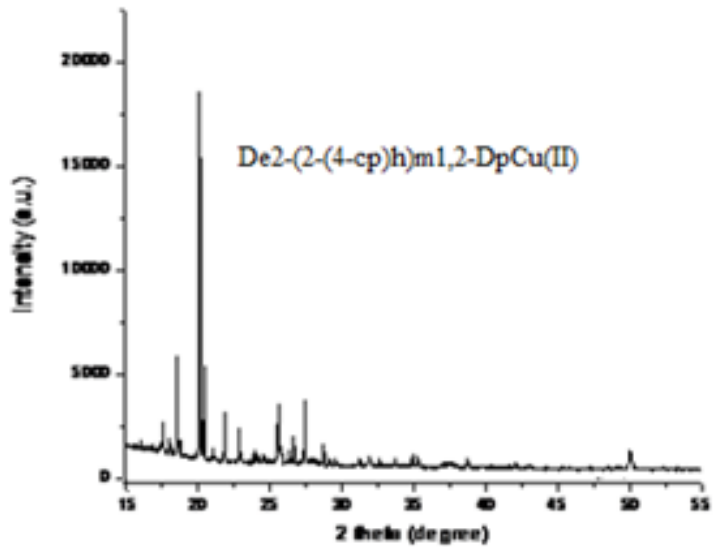

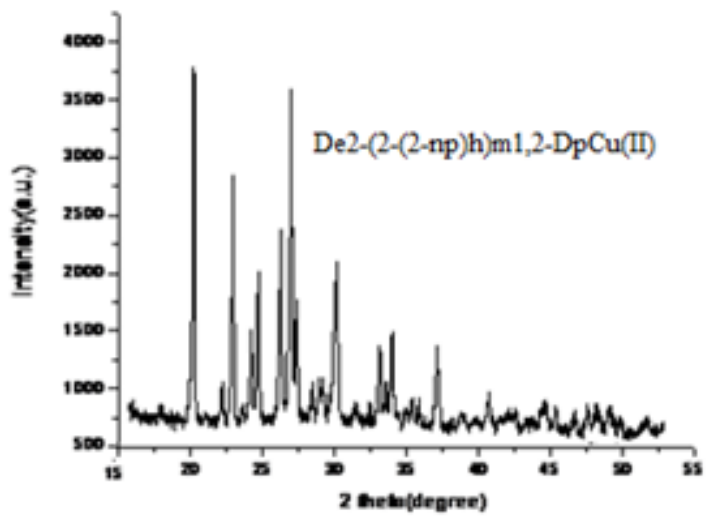

Figure 3. XRD pattern for $\mathrm{Cu}$ complexes
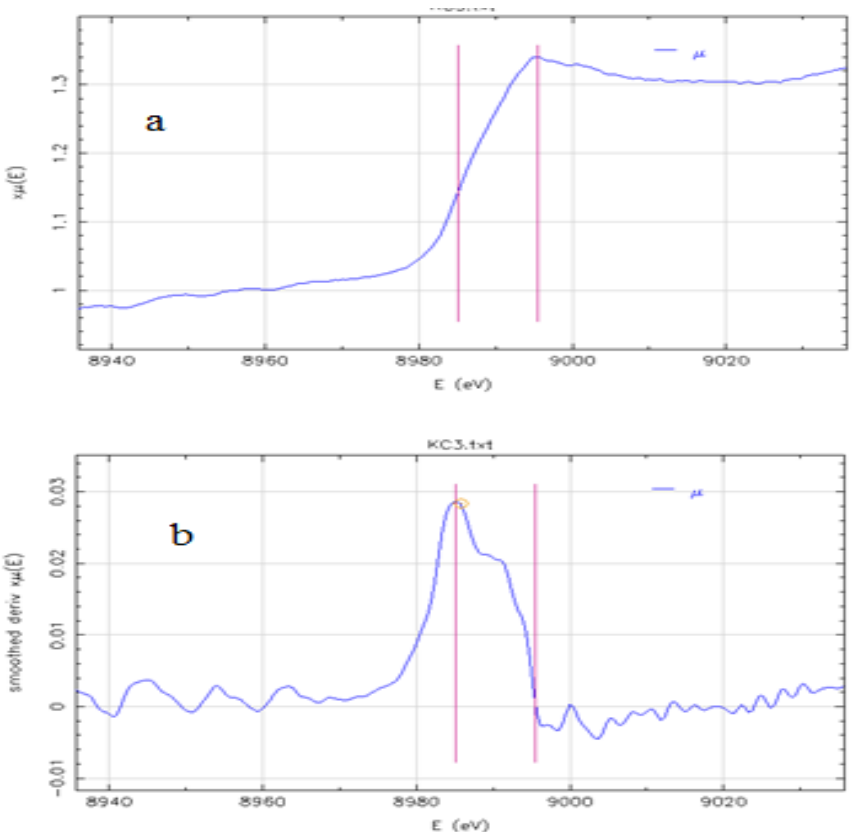

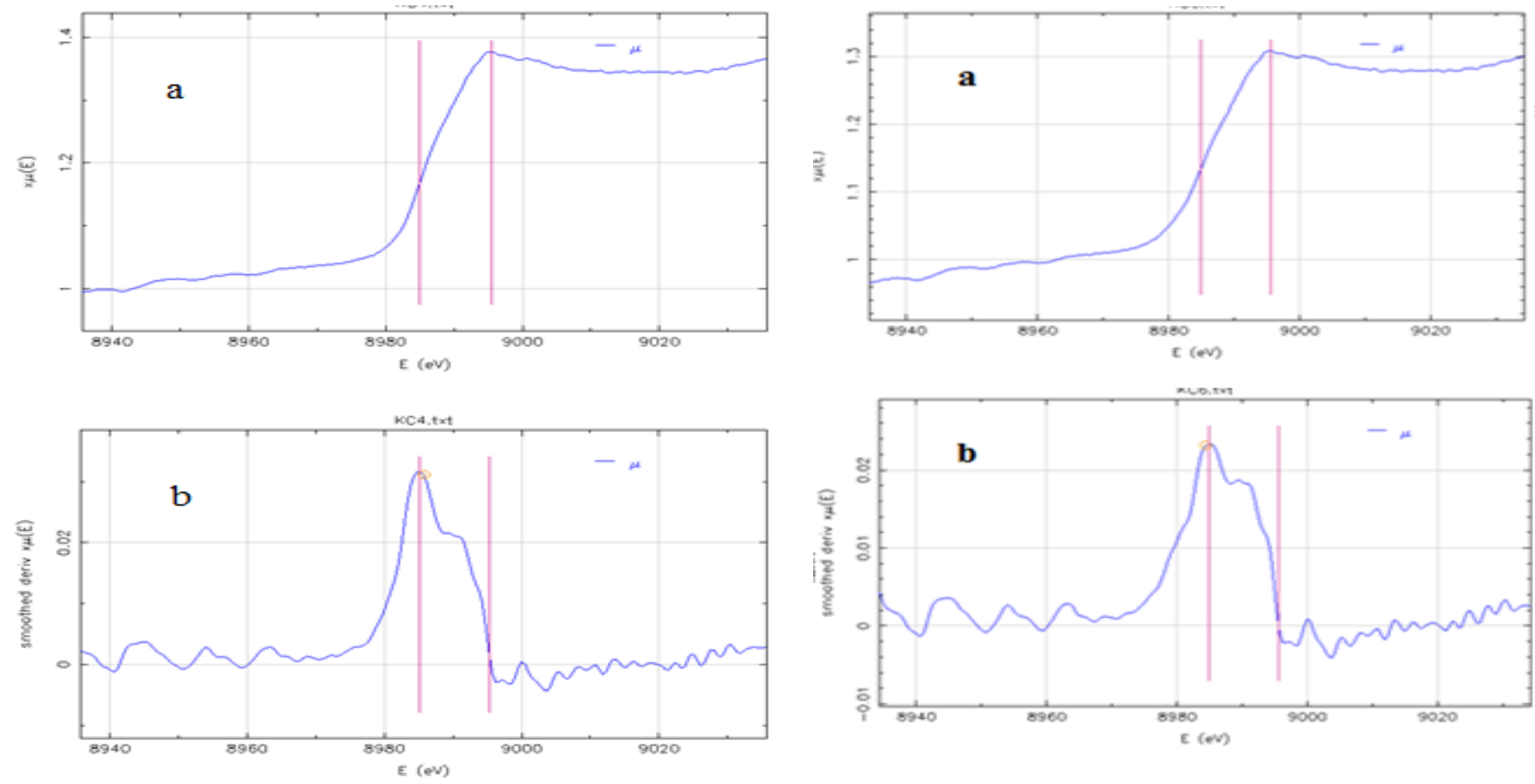

Figure 4. (a) XAFS spectrum and (b) Derivative of XAFS spectrum for copper metal indicating position of absorption edge K and principal absorption maxima for all metal complexes

Table 1. Particle size and lattice parameter

\begin{tabular}{|c|c|c|c|}
\hline \multicolumn{4}{|c|}{ Results of X-ray diffraction studies of Copper Complexes } \\
\hline S.No. & Complex name & Particle size(in $\mathbf{n m})$ & Lattice Parameter(in $\mathbf{n m})$ \\
\hline 1 & De2-(2-o-t)m1,2-DpCu(II) & 32.61 & 6.88 \\
\hline 2 & De2-(2-(4-cp)h)m1,2-DpCu(II) & 55.43 & 12.89 \\
\hline 3 & De2-(2-(2-np)h)m1,2-DpCu(II) & 52.87 & 13.40 \\
\hline S.No. & Complex name & Particle size(in $\mathbf{n m})$ & Lattice Parameter(in $\mathbf{n m})$ \\
\hline
\end{tabular}

Table 2 XANES data for K-absorption edge of Copper (II) complexes.

\begin{tabular}{|c|c|c|c|c|c|c|}
\hline S.No. & Complexes & EK (eV) & EA (eV) & $\begin{array}{c}\text { Chemical shift } \\
(\mathrm{eV})\end{array}$ & $\begin{array}{c}\text { Shift of the principal absorption maximum } \\
(\mathrm{eV})\end{array}$ & Edge-width(eV) \\
\hline 1 & Cu Metal & 8980.5 & 8995.69 & - & 14.96 & 15.19 \\
\hline 2 & $\begin{array}{c}\text { De2-(2-(3-Cp)h)m1,2- } \\
\text { DpCu(II) }\end{array}$ & 8985.91 & 8995.46 & 5.41 & 15.03 & 9.55 \\
\hline 3 & De2-(2-o-t)m1,2-DpCu(II) & 8985.88 & 8995.53 & 5.38 & 16.31 & 9.65 \\
\hline 4 & De2-(2-(4-cp)h)m1,2-DpCu(II) & 8986.28 & 8996.81 & 5.78 & 10.53 \\
\hline
\end{tabular}

\section{CONCLUSION}

On the basis of the above discussion, table1 Figure 3 shows the suggested structure for copper (II) complexes are octahedral and crystalline in nature. Range of particle size is $32.61-52-87 \mathrm{~nm}$ and range of lattice parameter is 6.88 to $13.40 \AA$. X-ray K-absorption near edge studies of all copper complexes suggests that the chemical shifts values are on the higher energy side. The values reported for copper complexes confirm that these complexes are ionic in nature. The edge width values are found to be on the high energy side. There is no splitting on the main edges of the complexes. These factors suggest the geometrical structure of the complexes to be octahedral.

\section{ACKNOWLEDGMENT}

The author is greatly thankful to Dr. M. Gupta, UGC-DAE Indore for allowing me to use the facility of XRD. Author is also thankful to Dr. S. N. Jha for taking measurements of $\mathrm{XAS}$, at RRCAT, Indore.

\section{REFERENCES}

[1] R.H. Holm, G.W. Everett, A. Chakravorty, "Prog. Inorg. Chem. 7” (1966) 83 
[2] W. Zhang, J.L. Loebach, S.R. Wilson, E.N. Jacobsen, "J. Am. Chem. Soc”. 112 (1990) 2801

[3] R.D. Jones, D.A. Summerville, F. Basolo, "Chem. Rev. "79 (1979)139

[4] A. Vogler, H. Kunkely, “Coord. Chem. Rev.” 177 (1998) 81.

[5] J.-L. Serrano, L. Oriol, “Adv. Mater." 7 (1995) 365.

[6] L. Mao, K. Yamamato, W. Zhou, L. "Jin, Electroanalysis" 12 (2000) 72.

[7] K. Chichak, U. Jacquembard, N.R. Branda, “Eur. J. Inorg. Chem." $8 \quad$ (2002) 357

[8] L.H. Vogt, H.M. Faigenbaum, S. Wiberly, “Chem. Rev. ” 63 (1963) 269

[9] S.J. Gruber, C.M. Harris, E. Sinn, “J. Inorg. Nucl. Chem.” 30 (1968) 1805

[10] Thompson K H, B“ohmerle K, Polishchuk E, Martins C, Toleikis P, Tse J, Yuen V,Mcneill J H and Orvig C 2004 "Journal of Inorganic Biochemistry" 982063

[11] Hothi H S, Makkar A, Sharma J R \& Manrao M R 2006 “Eur J Med Chem "41 253

[12] Zhang S, Tu C, and Wang X 2004 "European Journal of Chemistry" 984028

[13] Dutta S, Murugkar A, Gandhe N, and Padhye S 2001 "MetalBased Drugs" 8183

[14]E. A. Stern,in "x.ray absorption principles and applications, techniques of xafs, sexafs in addition,xanes"edited by (D. C. Koningsberger and Prins. New York. 1988) chi.

\section{AUTHORS PROFILE}

Mr. Kamaljeet Singh Sura pursuing Ph.D. under the supervision of Dr. Ashutosh Mishra Prof. and Head " School Of Physics" D.A.V.V. Indore (M.P.). The field of his research is condensed matter physics. He completed his M. Phil. from School of Physics and M. Sc. from Gov. Holkar Science Colllege. 